\title{
MARTIN BOUNDARY OF KILLED RANDOM WALKS ON ISORADIAL GRAPHS
}

\author{
CÉDRIC BOUTILLIER AND KILIAN RASCHEL, WITH AN APPENDIX BY ALIN BOSTAN
}

\begin{abstract}
We consider killed planar random walks on isoradial graphs. Contrary to the lattice case, isoradial graphs are not translation invariant, do not admit any group structure and are spatially non-homogeneous. Despite these crucial differences, we compute the asymptotics of the Martin kernel, deduce the Martin boundary and show that it is minimal. Similar results on the grid $\mathbb{Z}^{d}$ are derived in a celebrated work of Ney and Spitzer.
\end{abstract}

\section{Contents}

1. Introduction and results 1

2. Isoradial graphs, Green functions and exponential functions 5

3. Main results and proofs $\quad 10$

4. The case of periodic isoradial graphs 12

5. Minimal positive harmonic functions 18

Appendix A. Some thoughts on a positivity question (by Alin Bostan) 21

References 23

\section{Introduction and results}

1.1. Ney and Spitzer theorems. Let $\left(Z_{n}\right)_{n \geqslant 0}$ be a random walk on $\mathbb{Z}^{d}$ with finite support and non-zero drift. In the celebrated paper [47], Ney and Spitzer derive the asymptotics of the Green function

$$
G(x, y)=\sum_{n=0}^{\infty} \mathbb{P}_{x}\left[Z_{n}=y\right]
$$

as $|y| \rightarrow \infty$ in any given direction $\frac{y}{|y|} \rightarrow \hat{r} \in \mathbb{S}^{d-1}$, see [47, Thm 2.2]. Introduce the one-step transition probabilities generating function (with $\zeta \cdot y$ denoting the standard scalar product

Date: February 5, 2021.

2010 Mathematics Subject Classification. Primary 31C35, 05C81; Secondary 82B20, 60J45.

Key words and phrases. Martin boundary; Green function; isoradial graphs; killed random walk; discrete exponential function.

A. Bostan: Inria, Université Paris-Saclay, 1 rue Honoré d'Estienne d'Orves, 91120 Palaiseau, France.

C. Boutillier: Sorbonne Université, CNRS, Laboratoire de Probabilités, Statistique et Modélisation (UMR 8001), 4 Place Jussieu, F-75005 Paris, France \& Institut Universitaire de France.

K. Raschel: CNRS \& Institut Denis Poisson (UMR 7013), Université de Tours et Université d'Orléans, Parc de Grandmont, 37200 Tours, France.

This project has received funding from the European Research Council (ERC) under the European Union's Horizon 2020 research and innovation programme under the Grant Agreement No. 759702 and from the ANR DIMERS (ANR-18-CE40-0033). 
of $\zeta$ and $y$ in $\mathbb{R}^{d}$ )

$$
\phi(\zeta)=\sum_{y \in \mathbb{Z}^{d}} p(0, y) e^{\zeta \cdot y}
$$

defined in terms of the transition kernel $p(x, y)=\mathbb{P}_{x}\left[Z_{1}=y\right]$ of the random walk. Ney and Spitzer deduce from their asymptotics of (1) that

$$
\lim _{\substack{|y| \rightarrow \infty \\ y /|y| \rightarrow \hat{r}}} \frac{G\left(x_{1}, y\right)}{G\left(x_{0}, y\right)}=e^{\zeta(\hat{r}) \cdot\left(x_{1}-x_{0}\right),}
$$

where the mapping $\hat{r} \mapsto \zeta(\hat{r})$ is proved to define a homeomorphism between the ambient sphere $\mathbb{S}^{d-1}$ and the set

$$
\partial D=\left\{\zeta \in \mathbb{R}^{d}: \phi(\zeta)=1\right\},
$$

see [47, Cor. 1.3] and [30]; see also (18) for an expression of the above homeomorphism. Figure 1 contains a few examples of sets $\partial D$ in (4).

Introduce now the discrete Laplacian $\Delta=\mathrm{Id}-p$, i.e., for $x \in \mathbb{Z}^{d}$,

$$
\Delta f(x)=\sum_{y \in \mathbb{Z}^{d}} p(x, y)(f(x)-f(y)) .
$$

From a potential theory viewpoint it is obvious that the exponential functions $f(x)=e^{\zeta \cdot x}$ are discrete harmonic (meaning $\Delta f=0$ ) if and only if $\zeta \in \partial D$. It is further known $[17,20]$ that they are (positive) minimal, in the sense that if $h$ is harmonic and satisfies $0 \leqslant h \leqslant f$ on $\mathbb{Z}^{d}$, then $h=c f$ for some $0 \leqslant c \leqslant 1$.

A corollary of Ney and Spitzer result (3) is that the Martin compactification of the random walk coincides with the geometric compactification of $\mathbb{Z}^{d}$ by the sphere $\mathbb{S}^{d-1}$ at infinity. More precisely, any harmonic function $f$ for $\left(Z_{n}\right)_{n \geqslant 0}$ may be written as $f(x)=\int_{\partial D} e^{\zeta \cdot x} \mu(\mathrm{d} \zeta)$, where $\mu$ is a positive Radon measure on $\partial D$, see [47, Rem. a]. For a general introduction on Martin boundary see $[45,19,31,50,56]$.

Let us mention a few strongly related results. First, the original Ney and Spitzer result (3) does not assume the boundedness of the support (it is only assumed that every point of $\partial D$ has a neighborhood in which $\phi$ in (2) is finite), but throughout this article we shall only consider random walks with bounded increments. In absence of drift and if $d \geqslant 3$ (so as to have a transient behavior), the Martin boundary is reduced to a single point, see [52, Sec. 26] and [56, (25.11) and (25.12)] for exact statements and proofs. Let us also point out the concept of $t$-Martin boundary (which probabilistically corresponds to a random walk with killing), which involves the Green function

$$
G\left(x, y \mid \frac{1}{t}\right)=\sum_{n=0}^{\infty} \mathbb{P}_{x}\left[Z_{n}=y\right] \frac{1}{t^{n}} .
$$

Obviously $G(x, y \mid 1)=G(x, y)$ in our notation (1). This $t$-Martin boundary is either empty (when $t<\rho$, where $\rho$ is the spectral radius of the transition kernel), reduced to one point $(t=\rho)$ or homeomorphic to $\mathbb{S}^{d-1}$ (when $\left.t>\rho\right)$, see Woess [56, Sec. 25.B]. The ratio $G\left(x_{1}, y \mid \frac{1}{t}\right) / G\left(x_{0}, y \mid \frac{1}{t}\right)$ converges to the same quantity as in (3), but with $\zeta(\hat{r}) \in \partial D_{t}$, where

$$
\partial D_{t}=\left\{\zeta \in \mathbb{R}^{d}: \phi(\zeta)=t\right\} .
$$

We finally mention the work [23], in which Dussaule obtains the Martin boundary of thickenings of $\mathbb{Z}^{d}$ (i.e., Cartesian products of $\mathbb{Z}^{d}$ by a finite set). In the reference [3] Babillot considers non-lattice random walks and obtains the Martin boundary of thickenings of $\mathbb{R}^{d}$. 


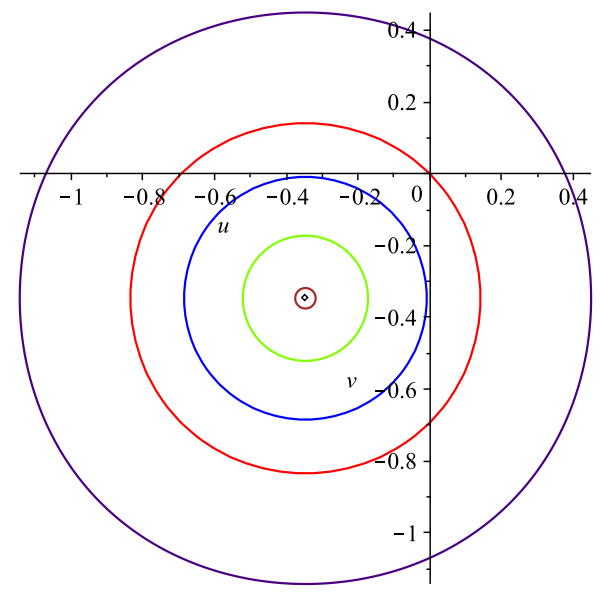

Figure 1. The curve $\phi\left(\zeta_{1}, \zeta_{2}\right)=t$, for $\phi\left(\zeta_{1}, \zeta_{2}\right)=\frac{e^{\zeta_{1}}}{3}+\frac{e^{\zeta_{2}}}{3}+\frac{e^{-\zeta_{1}}}{6}+\frac{e^{-\zeta_{2}}}{6}$ and $t=2 \sqrt{2} / 3 \approx 0.9428,0.943,0.95,0.97,1,1.1$. It corresponds to a nearest neighbor walk in $\mathbb{Z}^{2}$ with jump probability $\frac{1}{3}$ to the North and East, and $\frac{1}{6}$ to the South and West. The spectral radius of the transition kernel is $2 \sqrt{2} / 3$.

1.2. Spatial inhomogeneity. A crucial ingredient in the proof of all above results is the spatial homogeneity (or invariance by translation) of $\mathbb{Z}^{d}$ and of the random walk, meaning that $p(x, y)=p(0, y-x)$ for all $x, y \in \mathbb{Z}^{d}$.

The perturbation of the homogeneity at a finite number of sites of the grid $\mathbb{Z}^{d}$ should not affect the structure of the Martin boundary, but already impacts on the expressions for harmonic functions. This is shown by Kurkova and Malyshev [42, Thm 2.1] in the case of planar random walks with jumps to the nearest neighbors; they obtain the asymptotics of the Green function and show that the Martin boundary is homeomorphic to the circle $\mathbb{S}^{1}$. On the other hand, in the case of a homogeneity perturbed at an infinite number of points, computing the Green function asymptotics or deriving the Martin boundary seems too difficult in general (even if some upper and lower bounds may exist for the transition probabilities or for the Green functions, see [46]).

It is therefore natural to introduce a structure behind the inhomogeneities. A class of such models is composed of random walks killed or reflected when they exit a domain (inhomogeneities then appear on the boundary of the domain). In this context, Kurkova and Malyshev [42], Ignatiouk-Robert [33, 34, 35], Ignatiouk-Robert and Loree [32] obtain the asymptotics of (ratios of) Green functions as in (3) and derive the Martin boundary when the domain is a quarter plane $\mathbb{Z}_{+}^{2}$ or a half-space $\mathbb{Z}_{+} \times \mathbb{Z}^{d-1}$. See also [27].

1.3. The case of isoradial graphs. In this article we explore another class of models having inhomogeneities in infinite number, namely, killed random walks on isoradial graphs. By definition, isoradial graphs (see Figure 2 for a few examples) are planar embedded graphs such that all faces are inscribable in a circle of radius 1 ; more details will be provided in Section 2. So the irregularities of such graphs are structured, but as it is illustrated by looking at the pictures, the behavior may be quite wild. In particular, with the exception of a few simple particular cases (the square lattice $\mathbb{Z}^{2}$ or the triangular lattice), an isoradial graph is not a linear transform of a two-dimensional lattice, is not translation-invariant and can be actually highly non-homogeneous. Introduced by Duffin [21], Mercat [44] and Kenyon [39], 
these graphs have become particularly popular, as they are well suited for discrete complex analysis [16]. Moreover, because the star-triangle transformation (see Figure 4), which plays an important role in integrable statistical mechanics, preserves isoradiality, many models of statistical mechanics are now studied [12] on isoradial graphs: dimer model [39, 18], two-dimensional Ising model [14], spanning trees, bond percolation [28], random cluster model $[4,22]$, etc.

Despite their intrinsic irregularity and the absence of group structure, isoradial graphs form a broad class of graphs on which one can define an exponential function in a uniform way; this is a first step towards a Ney and Spitzer theorem. Such an exponential function is only proved to exist when the isoradial graph $\mathrm{G}$ is equipped with certain natural conductances, involving trigonometric or elliptic functions and geometric angles of the embedding of the graph. The existence is obtained by Kenyon [39] for critical (trigonometric) weights and is extended to the non-critical (elliptic) case by de Tilière and the two present authors [13]. Whereas the classical exponential function between any two points $x, y \in \mathbb{Z}^{d}$ may be written as $e^{\zeta \cdot(y-x)}$ with a parameter $\zeta \in \mathbb{C}$, see (3), the exponential function on $G$ takes the form

$$
\mathrm{e}_{(x, y)}(u \mid k) \text {, }
$$

where $x, y \in \mathrm{G}, k \in(0,1)$ is an elliptic modulus naturally attached to the model and $u$ lies on the torus $\mathbb{T}(k)=\mathbb{C} /\left(4 K(k) \mathbb{Z}+4 i K^{\prime}(k) \mathbb{Z}\right), K$ and $K^{\prime}$ denoting the elliptic integrals of the first kind and its complementary, respectively, see Section 2.3 and $[13$, Sec. 3.3]. In the same manner that $f(x)=e^{\zeta \cdot x}$ is harmonic on $\mathbb{Z}^{d}$ for $\zeta \in \partial D$, the exponential function $\mathrm{e}_{(x, y)}(u \mid k)$ is discrete harmonic as a function of $x$ ( $y$ being fixed), in the sense that its massive Laplacian

$$
\Delta f(x)=\sum_{z \sim x} \rho\left(\bar{\theta}_{x z} \mid k\right)(f(x)-f(z))+m^{2}(x \mid k) f(x)
$$

is zero by [13, Prop. 11], the conductances $\rho$ and (squared) masses $m^{2}$ being defined in (11) and (12). The work [13] also provides exact and asymptotic expressions for the Green function $G(x, y)$ of the killed random walk described by (8), see in particular [13, Thm 14].

1.4. Main results. Building on the results of [13], our main theorem is the asymptotics of the Martin kernel

$$
\lim _{y \rightarrow \infty} \frac{G\left(x_{1}, y\right)}{G\left(x_{0}, y\right)}=\mathrm{e}_{\left(x_{0}, x_{1}\right)}\left(u_{0} \mid k\right)
$$

where the exponential function $\mathrm{e}_{\left(x_{0}, x_{1}\right)}$ in $(7)$ is evaluated at a point $u_{0} \in 2 i K^{\prime}+\mathbb{R}$ which depends on the way that $y$ goes to infinity in the graph. See Theorem 1 for the precise statement. Let us present the main features of (9):

- First, it gives the limit of the ratio of the Green function in any asymptotic direction in the graph. It also proves that the Martin boundary is homeomorphic to the sphere $\mathbb{S}^{1}$, providing the announced generalization of Ney and Spitzer result (3) on isoradial graphs. The latter result follows from showing that $u_{0}$ describes a circle in the torus, as the asymptotic direction varies along all possible directions in the graph (see our Theorem 2).

- Theorem 3 shows that for all $u_{0} \in 2 i K^{\prime}+\mathbb{R}$ and $y \in \mathrm{G}, \mathrm{e}_{(x, y)}\left(u_{0} \mid k\right)$ defines a minimal positive harmonic function (see Section 1.1 for the definition), thereby proving that the Martin boundary is minimal. Recall that positive harmonic functions are particularly important in potential theory, as they allow, via the Doob transformation, 
to construct conditioned processes (for example going to infinity along a given direction in the graph).

- In the few particular cases which are both isoradial graphs and lattices (namely, the square and triangular lattices), we show that our results coincide with the classical Ney and Spitzer theorem and we unify the two points of view.

- Beyond the square and triangular lattices, our results in particular apply to periodic isoradial graphs, which are constructed as lifts to the plane of a fundamental pattern on the torus. We prove that some tools developed for their study (characteristic polynomial, amoeba, quantity $u_{0}$ in (9), etc.) naturally correspond with objects in $\mathbb{Z}^{d}$ (generating function of the transition probabilities, set $\partial D_{t}$ in (6), mapping $\zeta(\hat{r})$ in (3), etc.).

The paper is organized as follows. Section 2 presents some crucial definitions and properties of isoradial graphs, Green functions and discrete exponential functions. Section 3 contains the statements of the main results and their proofs. After gathering and reinterpreting existing results on general periodic planar graphs, Section 4 presents a refined study of the case of periodic isoradial graphs. Section 5 focusses on minimal harmonic functions.

Acknowledgments. We warmly thank Béatrice de Tilière for interesting discussions. We also thank an anonymous referee for his/her reading and comments.

\section{Isoradial graphs, Green functions and exponential functions}

2.1. Isoradial graphs. Isoradial graphs, named after [39], are defined as follows ${ }^{1}$. An embedded planar graph $\mathrm{G}=(\mathrm{V}, \mathrm{E})$ is isoradial if all internal faces are inscribable in a circle, with all circles having the same radius (fixed for example to 1 ), and such that all circumcenters are in the interior of the faces. Examples are provided in Figures 2 and 7.

Let $\mathrm{G}$ be an infinite, isoradial graph, whose bounded faces fill the whole plane. Then the dual graph $\mathrm{G}^{*}$, embedded by placing dual vertices at the circumcenters of the corresponding faces, is also isoradial.

The diamond graph, denoted $\mathrm{G}^{\diamond}$, is constructed from an isoradial graph $\mathrm{G}$ and its dual $\mathrm{G}^{*}$. Vertices of $G^{\diamond}$ are those of $G$ and those of $G^{*}$. A dual vertex of $G^{*}$ is joined to all primal vertices on the boundary of the corresponding face. Since edges of the diamond graph $\mathrm{G}^{\diamond}$ are radii of circles, they all have length 1 and can be assigned a direction $\pm e^{i \bar{\alpha}}$. Note that faces of $\mathrm{G}^{\diamond}$ are side-length 1 rhombi.

A train-track is a bi-infinite path on the dual of the diamond graph $\left(G^{\diamond}\right)^{*}$ which does not turn: when entering in a rhombus, it exits through the opposite edge. See Figure 2. A train-track $T$ thus crosses edges with the same direction $\pm e^{i \bar{\alpha}}$. We then say that the angle $\bar{\alpha}$ is associated to the train-track. This angle is a priori well defined only modulo $\pi$. This ambiguity can be lifted by considering an orientation for the train-track and choosing a convention (i.e., the vector $e^{i \bar{\alpha}}$ crosses the oriented train-track from left to right), and then, the angle associated to the train-track oriented in the other direction is $\bar{\alpha}+\pi$.

A minimal path between two vertices $x$ and $y$ is a shortest path in $G^{\diamond}$ between these vertices. It is not unique, but the set of unit steps used is the same for all minimal paths.

Using the diamond graph, angles can naturally be assigned to edges of the graph $G$ as follows. Every edge $e$ of $G$ is the diagonal of exactly one rhombus of $G^{\triangleright}$, and we let $\bar{\theta}_{e}$ be the half-angle at the vertex it has in common with e, see Figure 3. Note that we have

\footnotetext{
${ }^{1}$ The presentation of Section 2.1 follows that of [13, Sec. 2].
} 

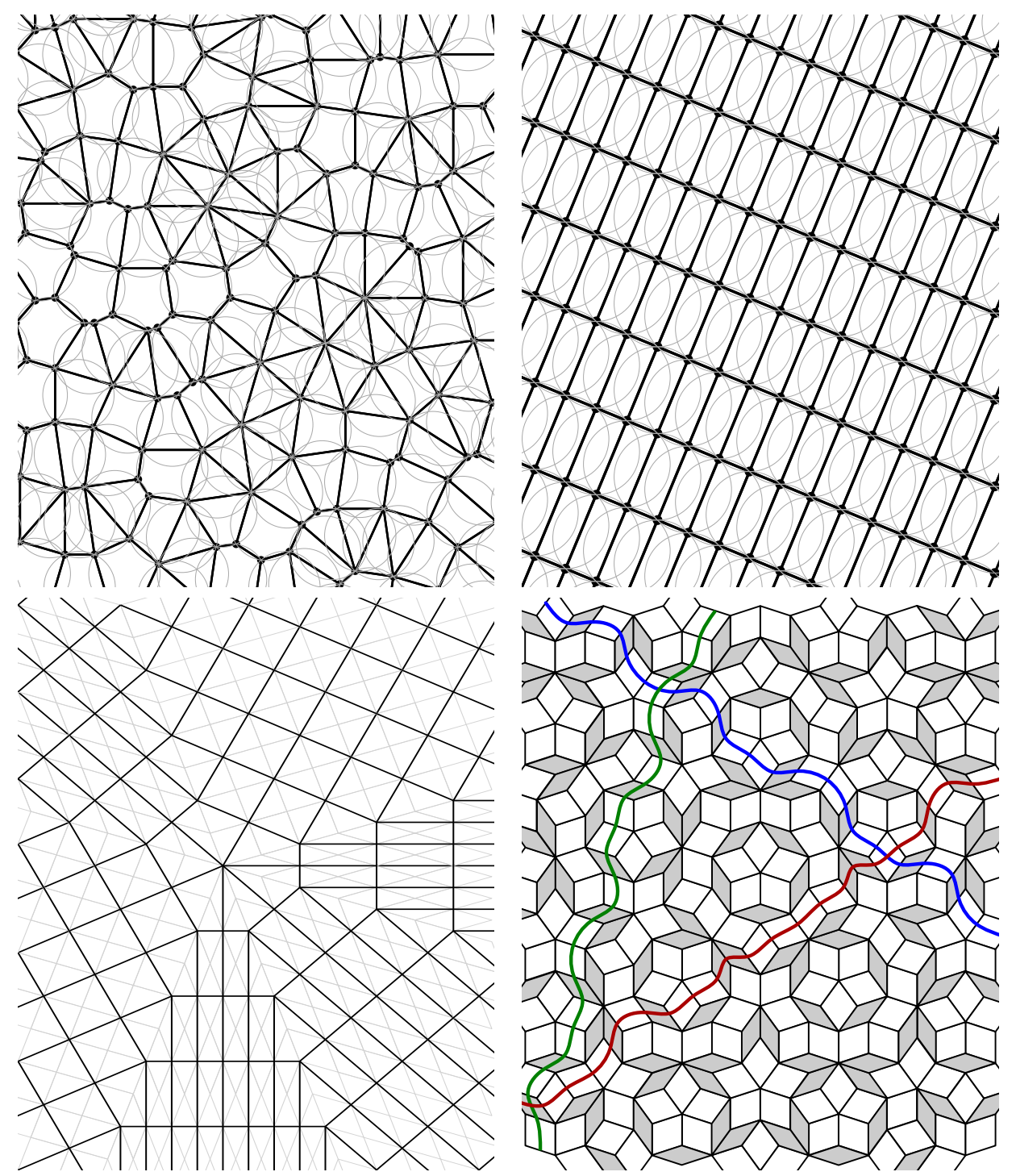

Figure 2. Upper left: piece of an infinite graph $\mathrm{G}$ isoradially embedded in the plane with the circumcircles of the faces. Upper right: a periodic isoradial embedding of the square lattice. Lower left: an isoradial graph with multiple cone-like regions (see Section 3.3 for a related discussion). Lower right: a piece of the Penrose tiling with rhombi, which can be used as the diamond graph of an isoradial graph, and three train-tracks, as bi-infinite paths in the dual.

$\bar{\theta}_{e} \in\left(0, \frac{\pi}{2}\right)$, because circumcircles are assumed to be in the interior of the faces. From now on, we actually ask more and suppose that there exists $\varepsilon>0$ such that $\bar{\theta}_{e} \in\left(\varepsilon, \frac{\pi}{2}-\varepsilon\right)$. We also assign two rhombus vectors to the edge $e$, denoted $e^{i \bar{\alpha}_{e}}$ and $e^{i \bar{\beta}_{e}}$, see Figure 3 , and we assume that $\bar{\alpha}_{e}, \bar{\beta}_{e}$ satisfy $\frac{\bar{\beta}_{e}-\bar{\alpha}_{e}}{2}=\bar{\theta}_{e}$.

An isoradial graph $G$ is said to be quasicrystalline if the number $d$ of possible directions assigned to edges of its diamond graph $G^{\diamond}$ is finite. 


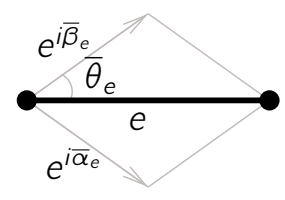

Figure 3. An edge $e$ of $G$ is the diagonal of a rhombus of $\mathrm{G}^{\triangleright}$, defining the angle $\bar{\theta}_{e}$ and the rhombus vectors $e^{i \bar{\alpha}_{e}}$ and $e^{i \bar{\beta}_{e}}$. One has $\frac{\bar{\beta}_{e}-\bar{\alpha}_{e}}{2}=\bar{\theta}_{e}$.

2.2. Monotone surfaces and asymptotically flat isoradial graphs. In the quasicrystalline case, the diamond graph can be seen as the projection of a monotone surface in $\mathbb{Z}^{d}$ onto the plane, and every edge of $G^{\diamond}$ is the projection of a unit vector of $\mathbb{Z}^{d}$, see $[15,6]$. Vertices (resp. faces) of $G$ correspond then to even (resp. odd) vertices of $\mathbb{Z}^{d}$ on that surface. For example, in the particular case when $d=3$, the diamond graph appears naturally to the eye as the projection in the plane of a landscape made of unit cubes, see for example Figure 7. Also, the Penrose tiling (see Figure 2, lower right) is the projection of a monotone surface very close to a given plane with irrational slope in $\mathbb{Z}^{5}$ [15].

Let $\left(e_{1}, e_{2}, \ldots, e_{d}\right)$ be the canonical basis of $\mathbb{Z}^{d}$ and $\bar{\alpha}_{1}, \bar{\alpha}_{2}, \ldots, \bar{\alpha}_{d}$ be the angles associated to the train-tracks such that $e^{i \bar{\alpha}_{j}}$ is the projection of $e_{j}$, and

$$
\bar{\alpha}_{1}<\bar{\alpha}_{2}<\cdots<\bar{\alpha}_{d}<\bar{\alpha}_{1}+\pi<\cdots<\bar{\alpha}_{d}+\pi<\bar{\alpha}_{1}+2 \pi .
$$

Using this notion of surface and the notation above, we can give a sense to the difference of two vertices $y-x$ as the vector $\sum_{j=1}^{d} N_{j} e_{j}$ in $\mathbb{Z}^{d}$ joining the corresponding points on the surface. The quantity

$$
N=|y-x|=\sum_{j=1}^{d}\left|N_{j}\right|
$$

is the graph distance between $x$ and $y$ seen as vertices of $\mathrm{G}^{\diamond}$, as well as the graph distance in $\mathbb{Z}^{d}$ between the corresponding points on the monotone surface. The reduced coordinates of $y-x$ are the quantities $n_{j}=\frac{N_{j}}{N}$, which define a point $\check{r}=\sum_{j=1}^{d} n_{j} e_{j}$ in the $L^{1}$-unit ball of $\mathbb{R}^{d}$.

Monotone surfaces can be wild in the following sense: fix a reference vertex $x_{0}$ and a direction $\hat{r} \in \mathbb{S}^{1}$, and look at the reduced coordinates $n_{j}$ in a minimal path from $x_{0}$ to $y$, as $y$ tends to infinity in the direction $\hat{r} \in \mathbb{S}^{1}$ in the plane embedding. These quantities may not converge. This is what happens for example on Figure 7 , because of the larger and larger "waves" causing oscillations for the $n_{j}$ 's.

We say that the isoradial graph is asymptotically flat if this does not happen, and if proportions converge for each directional limit. This property, and the values of the limits when they exist, do not depend on the choice of $x_{0}$. Periodic isoradial graphs and multiple cone-like regions (see Figure 2 and Section 3.3) are asymptotically flat. Let us call $n_{j}(\hat{r})$ the limits for $j=1, \ldots, d$ of the reduced coordinates in the direction $\hat{r}$. In that case, the function

$$
\hat{r} \mapsto \vec{n}(\hat{r})=\left(n_{j}(\hat{r})\right)_{1 \leqslant j \leqslant d}
$$

is automatically continuous and injective, as the map from the diamond graph to the monotone surface in $\mathbb{Z}^{d}$ is a bi-Lipschitz bijection. 
2.3. Exponential functions. We will be using definitions and results on the Z-invariant massive Laplacian $\Delta: \mathbb{C}^{\mathrm{V}} \rightarrow \mathbb{C}^{\mathrm{V}}$ introduced in $[13]^{2}$. Let $x$ be a vertex of $\mathrm{G}$ of degree $n$; denote by $e_{1}, \ldots, e_{n}$ the edges incident to $x$ and by $\bar{\theta}_{1}, \ldots, \bar{\theta}_{n}$ the corresponding rhombus half-angles, then the Laplacian operator is given by (8) (see [13, Eq. (1)] for the original statement), where the conductances $\rho$ and (squared) masses $m^{2}$ are defined by

$$
\begin{aligned}
\rho_{e}=\rho\left(\bar{\theta}_{e} \mid k\right) & =\operatorname{sc}\left(\theta_{e} \mid k\right), \\
m^{2}(x \mid k) & =\sum_{j=1}^{n}\left(\mathrm{~A}\left(\theta_{j} \mid k\right)-\operatorname{sc}\left(\theta_{j} \mid k\right)\right),
\end{aligned}
$$

where sc is one of the twelve Jacobi elliptic functions (see [1, Chap. 16] or [43, Chap. 2] for extensive presentations, or [13, App. A] for a selection of key properties) and

$$
\mathrm{A}(u \mid k)=\frac{1}{k^{\prime}}\left(\mathrm{Dc}(u \mid k)+\frac{E-K}{K} u\right),
$$

where $k^{\prime}=\sqrt{1-k^{2}}$ is the complementary elliptic modulus, $\operatorname{Dc}(u \mid k)=\int_{0}^{u} \mathrm{dc}^{2}(v \mid k) \mathrm{d} v$, and $E=E(k)$ is the complete elliptic integral of the second kind. Since $k$ is fixed once and for all, most of the time we will drop the elliptic modulus from our notation, writing for example $A(u)$ instead of $A(u \mid k)$.

We also need the definition of the complex-valued discrete $k$-massive exponential function $\mathrm{e}_{(x, y)}(u)$ of $[13$, Sec. 3.3], briefly introduced in (7), depending on a pair of vertices $(x, y)$ and of a complex parameter $u$. Consider an edge-path $x=x_{1}, \ldots, x_{n}=y$ of the diamond graph $\mathrm{G}^{\diamond}$ from $x$ to $y$ and let $e^{i \bar{\alpha}_{j}}$ be the vector corresponding to the edge $x_{j} x_{j+1}$. Then the exponential function is defined inductively along the edges of the path:

$$
\begin{aligned}
\forall u \in \mathbb{C}, \quad \mathrm{e}_{\left(x_{j}, x_{j+1}\right)}(u) & =i \sqrt{k^{\prime}} \operatorname{sc}\left(\frac{u-\alpha_{j}}{2}\right), \\
\mathrm{e}_{(x, y)}(u) & =\prod_{j=1}^{n-1} \mathrm{e}_{\left(x_{j}, x_{j+1}\right)}(u),
\end{aligned}
$$

where $\alpha_{j}=\bar{\alpha}_{j} \frac{2 K}{\pi}$. These functions do not depend on the path chosen for their definition, and are harmonic for the Laplacian (8), see [13, Prop. 11].

2.4. Exact and asymptotic expressions for the Green function. The massive Green function, denoted $G$, is the inverse of the massive Laplacian operator (8). The following formula is proved in [13, Thm 12]:

$$
G(x, y)=\frac{k^{\prime}}{4 i \pi} \int_{\Gamma_{x, y}} e_{(x, y)}(u) \mathrm{d} u,
$$

where $\Gamma_{x, y}$ is a vertical contour on the torus $\mathbb{T}(k)$, whose abscissa can be identified with the angle of the ray $\mathbb{R} \overrightarrow{x y}$. Let us remark that the discrete massive exponential function $\mathrm{e}_{(x, y)}(u)$ in (13) and (14) is defined using a path of the embedded graph from $x$ to $y$. This implies that the expression (14) for $G(x, y)$ is local, meaning that it remains unchanged if the isoradial graph $G$ is modified away from a path from $x$ to $y$. This is far from being a general situation: when computing the inverse of a discrete operator, one expects the geometry of the whole graph to be involved. The idea of the proof of the local formula (14) is the following: find a one-parameter family of local, complex-valued functions in the kernel

\footnotetext{
${ }^{2}$ The presentation of Section 2.3 follows that of [13, Sec. 3].
} 
of the massive Laplacian (8) (the exponential functions), define its inverse (minus the Green function) as a contour integral of these functions, and adjust (if possible) the contour of integration in such a way that $\Delta G=-$ Id.

Let us also state [13, Thm 14], which contains the asymptotic behavior of the Green function. Let $G$ be a quasicrystalline isoradial graph. Let $\chi$ the function defined (for $x$ and $y$ fixed) by

$$
\chi(v)=\chi(v \mid k)=\frac{1}{|x-y|} \log \mathrm{e}_{(x, y)}\left(v+2 i K^{\prime}\right),
$$

where $|x-y|$ is the graph distance between $x$ and $y$.

By [11, Lem. 17] the set of zeros of $\mathrm{e}_{(x, y)}(u)$ is contained in an interval of length $2 K-2 \varepsilon$, for some $\varepsilon>0$. Let us denote by $\alpha$ the midpoint of this interval. When the distance $\left|x_{0}-y\right|$ between vertices $x_{0}$ and $y$ of $G$ is large, we have

$$
G\left(x_{0}, y\right)=\frac{k^{\prime} e_{\left(x_{0}, y\right)}\left(2 i K^{\prime}+v_{0}\right)}{2 \sqrt{2 \pi\left|x_{0}-y\right| \chi^{\prime \prime}\left(v_{0}\right)}}(1+o(1)),
$$

where $v_{0}$ is the unique $v \in \alpha+(-K+\varepsilon, K-\varepsilon)$ such that $\chi^{\prime}(v)=0$, see [13, Lem. 15], and $\chi\left(v_{0}\right)<0$. Let us briefly recall the main idea in [13] to derive the asymptotics (16). Starting from (15), we may reformulate (14) as

$$
G\left(x_{0}, y\right)=\frac{k^{\prime}}{4 i \pi} \int_{\Gamma_{x_{0}, y}} e^{\left|x_{0}-y\right| x(v)} \mathrm{d} v .
$$

Typically, as $\left|x_{0}-y\right| \rightarrow \infty$, one may analyse the above integral using the saddle-point method. Classically, the saddle point is a critical point, therefore $\chi^{\prime}(v)=0$.

2.5. Going to infinity in an isoradial graph and 3D-consistency. Let us first recall that by construction $[19,31,50,56]$, a sequence of points $y$ converges to a point in the Martin boundary if it exits any finite subset of the graph and if the ratio of Green functions

$$
\frac{G\left(x_{1}, y\right)}{G\left(x_{0}, y\right)}
$$

converges pointwise. While (16) provides the main term in the Green function asymptotics and is independent on how y goes to infinity in the graph, we thus need here a different, somehow more precise information.

In the classical Ney and Spitzer theorem (3), y goes to infinity along an angular direction, namely $\frac{y}{|y|} \rightarrow \hat{r} \in \mathbb{S}^{1}$. However, this simple geometric description will not work in the isoradial setting. Indeed, it can be shown (see the proof of [13, Thm 14]) that the convergence of $v_{0}$ appearing in (16) is equivalent to the convergence of the reduced coordinates, which as already discussed (see Section 2.2 and Figure 7 ) is not guaranteed by the angular convergence of $y$.

In our opinion, the best way to characterize the convergence of the Martin kernel is to use the concept of 3D-consistency [6], that we now introduce. The notion of harmonicity for our massive Laplacian (8) is compatible with the star-triangle transformation (see Figure 4), which corresponds in the monotone surface picture to pushing the surface along a 3-dimensional cube: let $G_{Y}$ and $G_{\nabla}$ be two isoradial graphs differing by a star-triangle transformation, such that $G_{Y}$ has an extra vertex $x_{0}$ of degree 3. If $f$ is a harmonic function on $G_{\nabla}$, there is a unique way to extend it at $x_{0}$ to make it harmonic on $G_{Y}$. This operation, together with its inverse corresponding to forgetting the value at $x_{0}$, realize a bijection between harmonic functions on $\mathrm{G}_{Y}$ and $\mathrm{G}_{\nabla}$; see [13, Prop. 8]. 

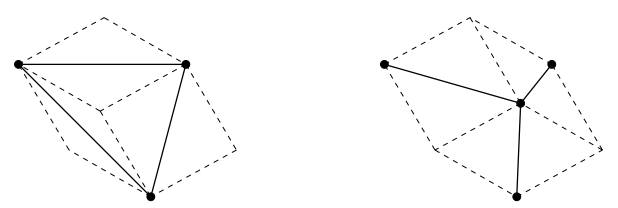

Figure 4. A star-triangle transformation on isoradial graphs. $G_{\nabla}$ is on the left, $G_{Y}$ on the right.

Using the 3D-consistency and the interpretation of quasicrystalline isoradial graphs as monotone surfaces on $\mathbb{Z}^{d}$ (Section 2.2), the exponential function and the Green function can be uniquely lifted to vertices of $\mathbb{Z}^{d}$, keeping their harmonicity properties. More precisely, if we assume that the isoradial graph $\mathrm{G}$ has enough train-tracks associated to each direction of $\mathbb{Z}^{d}$, namely that each coordinate on the monotone surface is not bounded either from above or below ${ }^{3}$, one can generate any monotone surface of $\mathbb{Z}^{d}$ from $G$ by a (maybe infinite) number of star-triangle transformations, and can extend the exponential and Green functions to all even vertices of $\mathbb{Z}^{d}$. Otherwise, one may get only half-spaces or slabs [7], but which are bi-infinite in $d^{\prime} \geqslant 2$ directions. We will often assume that we are in the first situation, in which $G$ spans all $\mathbb{Z}^{d}$. To extend our results to the second case, replacing $d$ by $d^{\prime}$ in the proofs will often be enough.

This lift to a lattice of higher dimension allows us to reformulate the asymptotics of the Green function as a limit as $y$ tends to infinity in a certain direction, thereby showing the strong parallel with the classical statement of Ney and Spitzer theorem. Indeed, in $\mathbb{Z}^{d}$ the convergence of $y$ along a direction is equivalent to the convergence of the reduced coordinates. These directions to infinity may not be obtained by staying on the surface corresponding to the graph $\mathrm{G}$ (unless for some directions, if the graph is asymptotically flat).

In the rest of the manuscript, we will always use this notion of convergence when we will compute the asymptotics of ratios of Green functions.

\section{Main results and proofs}

\subsection{Statements.}

Theorem 1 (asymptotics of the Martin kernel). Let $G$ be the massive Green function on a quasicrystalline isoradial graph, which can be lifted to $\mathbb{Z}^{d}$ via the star-triangle transformation. Then

$$
\lim _{y \rightarrow \infty} \frac{G\left(x_{1}, y\right)}{G\left(x_{0}, y\right)}=\mathrm{e}_{\left(x_{0}, x_{1}\right)}\left(u_{0}\right)
$$

where $u_{0}=2 i K^{\prime}+v_{0}$, with $v_{0}$ defined as in (16).

Theorem 2 (structure of the Martin boundary). As the asymptotic direction of $y \rightarrow \infty$ in (17) varies in $\mathbb{S}^{d-1}$, the point $u_{0}$ describes entirely the circle $2 i K^{\prime}+\mathbb{R} / 4 K \mathbb{Z}$ in $\mathbb{T}(k)$. If moreover $\mathrm{G}$ is asymptotically flat, the Martin boundary is homeomorphic to the circle $\mathbb{S}^{1}$.

Theorem 3 (minimality of the Martin boundary). Let $x_{0} \in \mathrm{G}$ be any fixed point in the graph and $v \in \mathbb{R} / 4 K \mathbb{Z}$. The positive harmonic functions $h_{v}(x)=\mathrm{e}_{\left(x_{0}, x\right)}\left(2 i K^{\prime}+v\right)$ are minimal.

\footnotetext{
${ }^{3}$ With the $n_{j}$ 's denoting the reduced coordinates of $y-x$, where $x$ is some reference vertex, this is equivalent to asking that for each $j$ and \pm , there is at least a direction $\hat{r}$ along which $\lim \sup \pm n_{j}$ is strictly positive as $y$ tends to infinity along $\hat{r}$, or in the planar embedding picture, asking that for any train-track $T$ associated to a direction $\alpha$, the two half-planes obtained by cutting along the parallel edges of $T$ have both an infinite number of train-tracks with this direction $\alpha$.
} 


\subsection{Proofs.}

Proof of Theorem 1. The starting point is the asymptotics (16) obtained in [13], that we first apply to the Green function $G\left(x_{0}, y\right)$. To derive the asymptotics of $G\left(x_{1}, y\right)$ we begin by rewriting (14) as

$$
G\left(x_{1}, y\right)=\frac{k^{\prime}}{4 i \pi} \int_{\Gamma_{x_{1}, y}} \mathrm{e}_{\left(x_{1}, x_{0}\right)}(u) \mathrm{e}_{\left(x_{0}, y\right)}(u) \mathrm{d} u .
$$

We then perform an asymptotic analysis of the above integral. The only difference with the proof of [13, Thm 14] is the presence of the term $\mathrm{e}_{\left(x_{1}, x_{0}\right)}(u)$, compare with (14). Using the saddle point method and similar ideas as in [13], we obtain that this factor will actually appear as a prefactor in the final asymptotics, evaluated at $u_{0}$. In other words,

$$
G\left(x_{1}, y\right)=\mathrm{e}_{\left(x_{1}, x_{0}\right)}\left(u_{0}\right) \frac{k^{\prime} \mathrm{e}_{\left(x_{0}, y\right)}\left(u_{0}\right)}{2 \sqrt{2 \pi\left|x_{0}-y\right| \chi^{\prime \prime}\left(v_{0}\right)}}(1+o(1))
$$

with the exact same $u_{0}=2 i K^{\prime}+v_{0}$ as in (16). We then easily reach the conclusion that the ratio of Green function behaves as in (17), which completes the proof.

Proof of Theorem 2. There is correspondence between the direction along which y goes to infinity and $u_{0}=2 i K^{\prime}+v_{0}$, see $\left[13\right.$, Lem. 15] and its proof. This $v_{0}$ can be anywhere on the circle $\mathbb{R} / 4 K \mathbb{Z}$ : the interval from the asymptotics of the Green function moves with the set of poles of the exponential function, which depends on the direction of $y$.

Now we assume $\mathrm{G}$ to be asymptotically flat, so the application $\hat{r} \mapsto \vec{n}(\hat{r})=\left(n_{j}(\hat{r})\right)_{1 \leqslant j \leqslant d}$ is well defined and continuous. It is even differentiable everywhere, except for $\hat{r}$ for which at least one of the $n_{j}(\hat{r})$ is zero (meaning that the corresponding minimal path seen on the monotone surface is moving to another orthant of $\mathbb{Z}^{d}$ ), but even at these points it has left and right derivatives.

We want to prove that $\hat{r} \mapsto u_{0}(\hat{r})$ is differentiable (has one-sided derivatives) when $\hat{r} \mapsto n_{j}(\hat{r})$ is differentiable (has one-sided derivatives), and that the derivative is strictly positive, in the sense that the points move in the same direction around their respective circles.

Up to a possible cyclic relabelling of the coordinates, we can assume that all the $n_{j}(\hat{r})$ are non-negative, and we look at a variation of $\hat{r}$ in the positive direction. Looking at how frequencies of steps along an infinite path should vary as the asymptotic direction in the plane moves slightly in the positive direction while the path stays in the same orthant, we see that the (one-sided) derivative $\frac{d \vec{n}}{d \hat{r}}$ of the reduced coordinates is a linear combination with positive coefficients of vectors of the form

$$
\delta n_{k, l}=(0, \ldots, 0,-1,0, \ldots, 0,1,0),
$$

where the 1 is at position I and the -1 at position $k<I$, namely,

$$
\frac{d \vec{n}}{d \hat{r}}=\sum_{k<l} a_{k, l}(\hat{r}) \delta n_{k, l},
$$

with $a_{k, l}(\hat{r}) \geqslant 0$, and not all equal to zero.

Rewrite the condition that $v_{0}=u_{0}-2 i K^{\prime}$ is a critical point as in the proof of [13, Lem. 15]:

$$
\chi^{\prime}\left(v_{0}\right)=\sum_{j=1}^{d} n_{j} \frac{\mathrm{sn} \cdot \mathrm{cn}}{\mathrm{dn}}\left(\frac{v_{0}-\alpha_{j}}{2}\right)=\vec{n} \cdot \vec{F}\left(v_{0}\right)=0
$$


with $\vec{F}(v)=\left(\frac{\mathrm{sn} \cdot \mathrm{cn}}{\mathrm{dn}}\left(\frac{v-\alpha_{j}}{2}\right)\right)_{1 \leqslant j \leqslant d}$. The implicit function theorem implies that $v_{0}$, as a function of $\hat{r}$, has a (one-sided) derivative $\frac{d v_{0}}{d \hat{r}}$, and it satisfies:

$$
\frac{d v_{0}}{d \hat{r}} \chi^{\prime \prime}\left(v_{0}\right)+\frac{d \vec{n}}{d \hat{r}} \cdot \vec{F}\left(v_{0}\right)=0
$$

Now, writing down explicitly the scalar product

$$
\frac{d \vec{n}}{d \hat{r}} \cdot \vec{F}\left(v_{0}\right)=\sum_{k<1} a_{k, l}\left(\frac{\mathrm{sn} \cdot \mathrm{cn}}{\mathrm{dn}}\left(\frac{v_{0}-\alpha_{l}}{2}\right)-\frac{\mathrm{sn} \cdot \mathrm{cn}}{\mathrm{dn}}\left(\frac{v_{0}-\alpha_{k}}{2}\right)\right),
$$

we see that this quantity is strictly negative, as all the quantities $\frac{v_{0}-\alpha_{j}}{2}$ are in the interval $[-K, K]$, on which the function $\frac{\mathrm{sn} \cdot \mathrm{cn}}{\mathrm{dn}}$ is strictly increasing (recall that $\alpha_{k}<\alpha_{l}$ ).

Since $v_{0}$ corresponds to a simple critical point of $\chi$, which is actually a local minimum, $\chi^{\prime \prime}\left(v_{0}\right)$ is strictly positive. As a consequence,

$$
\frac{d v_{0}}{d \hat{r}}>0
$$

This implies that the application

$$
T: \hat{r} \in \mathbb{S}_{1} \mapsto u_{0} \in 2 i K^{\prime}+\mathbb{R} / 4 K \mathbb{Z}
$$

is continuous, and lifts to a strictly increasing function on the universal cover. Moreover, we know [11, Lem. 17] that the interval of length $2 K-2 \varepsilon$ in which the real part of $u_{0}$ lies is winding once around the circle. Indeed, it should contain the zeros of the exponential function from the base vertex $x$ to $y$. When $y$ switches to the next orthant, while going around $x$, the sliding interval is dropping the $\alpha_{j}$ closer to its right boundary, and swallows $\alpha_{j}+\pi$. Therefore, after a full turn around $x$, the left and right boundaries of this sliding interval made also exactly one full turn. Since the total length between the left end of this interval at the beginning of the full turn and its right end at the end is strictly less than twice the length of the circle, it means that $T$ winds also exactly once around the circle, therefore $T$ is bijective. Since the image is compact, $T$ is automatically open, so it defines a homeomorphism.

Proof of Theorem 3. It will be given in Section 5.

3.3. An example: cones of homogeneities. The simplest inhomogeneous extension of the fully homogeneous case of $\mathbb{Z}^{d}$ consists in modifying the jumps at a finite number of sites, as explained in our introduction (see also [42]). Another simple extension is to split the grid in a finite union of connected domains, for example two half-planes, or more generally a finite number of cone-like regions as on Figure 2, and to assign to each region a (different) set of transition probabilities. Up to our knowledge, a Ney and Spitzer theorem is not known in this simple setting, even in the simplest instance of two half-planes. It is worth mentioning that our approach allows to study such cases, provided the transition probabilities follow the construction of elliptic conductances as introduced in Section 2.

\section{The case of periodic isoradial graphs}

In Section 4.1, we first study general periodic isoradial graphs and relate important quantities needed in their analysis (characteristic polynomial, amoeba, degeneracy of the amoeba, quantity $u_{0}$ in (9), etc.) to natural objects in $\mathbb{Z}^{d}$ (generating function of the transition probabilities, set $\partial D_{t}$ in (6), spectral radius, mapping $u(p)$ in (3), etc.). Then we 
show in Section 4.2 how our results give another proof of the classical Ney and Spitzer in two cases (the square and triangular lattices).

4.1. Amoeba of the characteristic polynomial and jump generating function. Using the theory of analytic combinatorics in several variables [49], we analyze the exponential decay of the Green function through Fourier analysis. We denote a vertex of $\mathrm{G}$ by a triple $\left(v, n_{1}, n_{2}\right)$, where $v$ is the copy of the vertex in the fundamental domain and $\left(n_{1}, n_{2}\right)$ is the element of $\mathbb{Z}^{2}$ corresponding to the translation moving $v$ to the vertex.

A Ney-Spitzer theorem for periodic planar graphs. The first part of this section is not specific to isoradial graphs and holds for all planar periodic graphs (having periodic masses and conductances) with at least one positive mass.

The Laplacian can be seen as a periodic convolution operator on vector-valued functions of $\mathbb{Z}^{2}$. It acts in Fourier space by multiplication by a matrix $\Delta(z, w)$, with $|z|=|w|=1$. The square matrix $\Delta(z, w)$ has rows and columns indexed by vertices of the fundamental domain, and the coefficient between $v$ and $w$ is the sum of the conductances of the edges between $(v, 0,0)$ and $\left(w, n_{1}, n_{2}\right)$ multiplied by $z^{n_{1}} w^{n_{2}}$.

If $\left(v, n_{1}, n_{2}\right)$ and $\left(w, n_{1}^{\prime}, n_{2}^{\prime}\right)$ are two vertices of $G$, the Green function can be expressed as the Fourier inverse transform of $\Delta(z, w)^{-1}=\frac{Q(z, w)}{P(z, w)}$, where $P(z, w)=\operatorname{det} \Delta(z, w)$ and $Q(z, w)$ is the adjugate matrix of $\Delta(z, w)$ :

$$
G\left(\left(v, n_{1}, n_{2}\right),\left(w, n_{1}^{\prime}, n_{2}^{\prime}\right)\right)=\iint_{|z|=|w|=1} \frac{z^{n_{1}-n_{1}^{\prime}} w^{n_{2}-n_{2}^{\prime}} Q(z, w)_{v, w}}{P(z, w)} \frac{\mathrm{d} z}{2 i \pi z} \frac{\mathrm{d} w}{2 i \pi w} .
$$

The asymptotic behavior of the Green function above is encoded in the singularities of the integrand, namely, the zeros of $P$. The zero-set of $P$ defines an algebraic curve, called the spectral curve of the Laplacian. The amoeba of $P$ (see [25, Chap. 6]) is the image of the spectral curve by the application

$$
(z, w) \mapsto(\log |z|, \log |w|)
$$

and plays an important role in the discussion; see Figure 5 for an example.

In the massive case, there is no zero of $P$ on the unit torus $|z|=|w|=1$, and thus we can deform continuously the contour of integration defining $G$ to increase or decrease the radii of the torus, until we touch the spectral curve, in order to obtain exponential decay.

We can say even more, because of geometric properties of spectral curves of Laplacians. The spectral curve is always a simple Harnack curve [40]. In particular, the boundary of the amoeba is the image of the real locus of the spectral curve. The bounded connected component of the complementary of the amoeba containing the origin (called the oval $\mathcal{O}$ in the sequel, see Figure 5) is a convex set whose boundary corresponds to a (subset) of positive real roots of $P$ :

$$
P\left(e^{\zeta_{1}}, e^{\zeta_{2}}\right)=0
$$

which in the classical theorem by Ney and Spitzer is the generalization of the level set $\partial D_{t}$ defined in (6). All conditions are met to be in the smooth point situation described in [49, Chap. 9], allowing us to readily obtain the asymptotics of the ratio of Green functions for copies of the same vertex $v$ in the fundamental domain. 


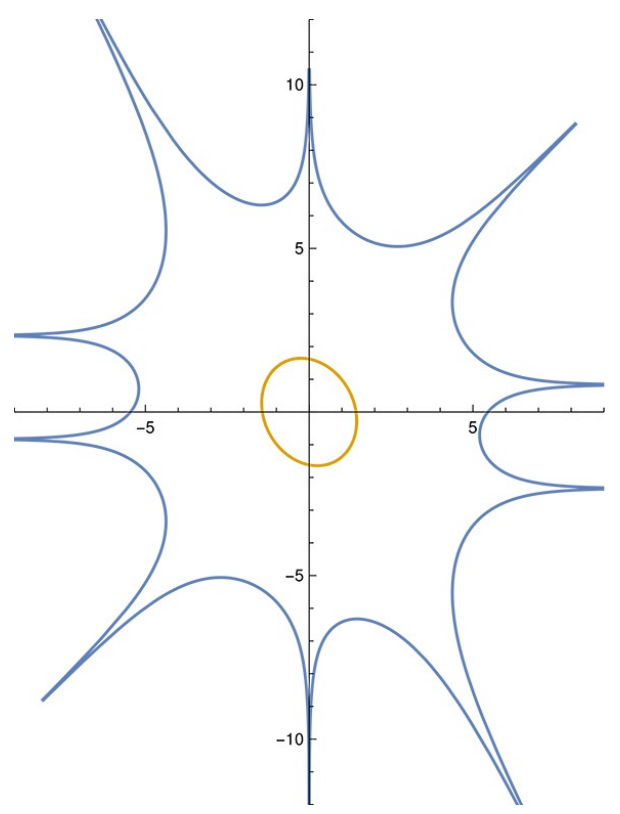

Figure 5. Amoeba of the spectral curve of a Laplacian with mass on a periodic graph. The amoeba itself is the region between the yellow and blue curves. The curve here has geometric genus one, and the (unique in this case) bounded connected component of the complementary of the amoeba containing the origin is called the oval.

Let $x_{0}=(v, 0,0), x_{1}=\left(v, n_{1}, n_{2}\right)$ and $y=\left(v, n_{1}^{\prime}, n_{2}^{\prime}\right)$. As $\left(n_{1}^{\prime}, n_{2}^{\prime}\right)$ goes to infinity in $\mathbb{Z}^{2}$ in the direction $\hat{r}$, we get the following asymptotics for the Green function:

$$
\lim _{y \rightarrow \infty} \frac{G\left(x_{1}, y\right)}{G\left(x_{0}, y\right)}=e^{\zeta \cdot n},
$$

where $\zeta=\zeta(\hat{r}):=\arg \max \{\hat{r} \cdot s: s \in \mathcal{O}\}$, which by convexity is reached along the boundary of $\mathcal{O}$. The homeomorphism $\psi: \hat{r} \in \mathbb{S}^{1} \mapsto \zeta(\hat{r}) \in \partial \mathcal{O}$ is the analogue of the Ney and Spitzer function $\zeta$ in the fully transitive case, which we recall here for completeness:

Lemma 4 ([30]). Let $\rho$ denote the spectral radius of the transition kernel. Assume $t \geqslant \rho$, with $t \neq \rho$ if the random walk has zero drift. Then the set $D_{t}=\left\{\zeta \in \mathbb{R}^{d}: \phi(\zeta) \leqslant t\right\}$ is compact and convex, the gradient

$$
\operatorname{grad} \phi(\zeta)=\sum_{x \in \mathbb{Z}^{d}} x e^{\zeta \cdot x} P(0, x)
$$

exists everywhere on $D_{t}$ and does not vanish on its boundary $\partial D_{t}$. Furthermore, the mapping

$$
\zeta \mapsto \hat{\mathrm{r}}=\frac{\operatorname{grad} \phi(\zeta)}{|\operatorname{grad} \phi(\zeta)|}
$$

determines a homeomorphism between $\partial D_{t}$ and $\mathbb{S}^{d-1}$.

The following result is stated under Corollary 1.3 in [47] for $t=1$ and random walks with drift; it is extended to other values of $t$ and to the zero drift case in [56, (25.21)]. 
Theorem 5. Under the same assumptions as in Lemma 4, let $\hat{r}$ be a point in $\mathbb{S}^{d-1}$ and $\zeta(\hat{r})$ be the unique solution in $\partial D_{t}$ of (18). Let $y$ denote any sequence in $\mathbb{Z}^{d}$ such that $|y| \rightarrow \infty$ and $\frac{y}{|y|} \rightarrow \hat{r}$. Then the ratio $G\left(x_{1}, y \mid \frac{1}{t}\right) / G\left(x_{0}, y \mid \frac{1}{t}\right)$ converges to $e^{\zeta(\hat{r}) \cdot\left(x_{1}-x_{0}\right)}$.

The isoradial periodic case. When in addition the periodic graph $\mathrm{G}$ is isoradial, with the elliptic conductances and masses from (11) and (12), the spectral curve has geometric genus 1 and there is a unique bounded connected component of the complementary of the amoeba [13]. Furthermore, we have an explicit parameterization of its boundary via the exponential function:

$$
\xi: u \mapsto\left(\log \left|e_{x, x+(1,0)}(u)\right|, \log \left|e_{x, x+(0,1)}(u)\right|\right),
$$

where $u \in 2 i K^{\prime}+\mathbb{R} / 4 K \mathbb{Z}$. The composition $\xi^{-1} \circ \psi$ is the application giving the $u_{0}$ from the asymptotics of Green function of Equation (16) for every direction $\hat{r}$ along which $y$ can go to infinity.

4.2. Examples. Among all periodic isoradial graphs, two of them (namely, the square and triangular lattices) are of special interest, as they correspond to models of homogeneous random walks in $\mathbb{Z}^{2}$. In this section we show how, in these two cases, our results match with the classical Ney and Spitzer theorem.

In order to state precisely this connection, it is important to notice that in Ney and Spitzer framework, a set of transition probabilities is fixed (through the function $\phi$ in (2)) and then the $t$-Martin boundary is computed for any fixed $t$ larger than the spectral radius. On the other hand, in the isoradial setting the conductances (related to the transition probabilities) and the mass (related to the variable $t$ ) both depend on the same elliptic modulus $k$, and it is a priori unclear that we can construct a random walk model with transition probabilities independent of the $t$ variable. This will be shown in the two above-mentioned examples, using the degrees of freedom that we have on the conductances and on the elliptic modulus.

The square lattice. The first example is the square lattice with angles $\alpha$ and $\beta$ associated to the two families of parallel train-tracks, with $\alpha<\beta<\alpha+2 K<\beta+2 K$, see Figure 2 . Because the definition of conductances and masses is invariant by rotation of the whole embedding, one can further assume that $\beta=-\alpha=\theta$ (see Figure 3). There are thus two types of edges: horizontal with opening angle $\bar{\theta}=\frac{\pi}{2 K} \theta \in\left(0, \frac{\pi}{2}\right)$ and vertical with opening angle $\frac{\pi}{2}-\bar{\theta}$. The characteristic polynomial of the model is given by $[13, \mathrm{Sec} .5 .2]$

$$
P(z, w)=m+2\left(c_{1}+c_{2}\right)-c_{1}\left(z+\frac{1}{z}\right)-c_{2}\left(w+\frac{1}{w}\right),
$$

with $c_{1}=\mathrm{sc}(\theta), c_{2}=\mathrm{sc}(K-\theta)$ and $m$ as in (12). It is convenient to normalize (20) so as to have transition probabilities summing to one:

$$
\widetilde{P}(z, w)=\frac{m+2\left(c_{1}+c_{2}\right)}{2\left(c_{1}+c_{2}\right)}-p_{1}\left(z+\frac{1}{z}\right)-p_{2}\left(w+\frac{1}{w}\right),
$$

with

$$
p_{1}=\frac{c_{1}}{2\left(c_{1}+c_{2}\right)} \quad \text { and } \quad p_{2}=\frac{c_{2}}{2\left(c_{1}+c_{2}\right)}=\frac{1}{2}-p_{1}
$$

It is natural to associate to this model the classical simple random walk on $\mathbb{Z}^{2}$, with transition probabilities Laplace transform

$$
\phi\left(\zeta_{1}, \zeta_{2}\right)=p_{1}\left(e^{\zeta_{1}}+e^{-\zeta_{1}}\right)+p_{2}\left(e^{\zeta_{2}}+e^{-\zeta_{2}}\right)
$$


Notice that horizontal (resp. vertical) jumps have the same weight, due to the constraints on the conductances in (20) (in particular, the random walk has zero drift). We now state precisely the connection between the two frameworks.

Transition probabilities generating function, characteristic polynomial and their zero-sets. It is clear that $\widetilde{P}(z, w)=0$ if and only if $\phi(\log z, \log w)=t$, with $t=1+m /\left(2 c_{1}+2 c_{2}\right)$. In particular, using the notation of Section 4.1, the oval of the amoeba is in correspondence with the set $\partial D_{t}$.

Exponential functions. As said in the introduction, extremal positive harmonic functions in group structures are exponential functions [17]. In our context of the planar lattice, they are given by (writing $\zeta=\left(\zeta_{1}, \zeta_{2}\right)$ and $\left.n=\left(n_{1}, n_{2}\right)\right)$

$$
f(x)=e^{\zeta \cdot n}=\left\{e^{\zeta_{1}}\right\}^{n_{1}}\left\{e^{\zeta_{2}}\right\}^{n_{2}},
$$

for $\zeta \in \partial D_{t}$. By definition of $\partial D_{t}$, see (6), the pairs $\left(e^{\zeta_{1}}, e^{\zeta_{2}}\right)$ parametrize the zero-set of $\phi-t$

To compare the elementary expression (22) for the exponential function to its elliptic analogue (13), we should first introduce an elliptic uniformisation of the zero-set of the characteristic polynomial (20). As shown in [13, Eq. (32)] (see also (19)),

$$
\left\{(z, w) \in(\mathbb{C} \cup\{\infty\})^{2}: P(z, w)=0\right\}=\{(z(u), w(u)): u \in \mathbb{T}(k)\},
$$

with

$$
z(u)=-k^{\prime} \operatorname{sc}\left(\frac{u-\alpha}{2}\right) \operatorname{sc}\left(\frac{u-\beta}{2}\right) \quad \text { and } \quad w(u)=\frac{\operatorname{sc}\left(\frac{u-\beta}{2}\right)}{\operatorname{sc}\left(\frac{u-\alpha}{2}\right)} .
$$

Moreover, using (20) one obtains

$$
\mathrm{e}_{\left(n_{1}, n_{2}\right)}(u)=\left\{i \sqrt{k^{\prime}} \mathrm{sc}\left(\frac{u-\alpha}{2}\right)\right\}^{n_{1}-n_{2}}\left\{i \sqrt{k^{\prime}} \mathrm{sc}\left(\frac{u-\beta}{2}\right)\right\}^{n_{1}+n_{2}}=z(u)^{n_{1}} w(u)^{n_{2}} .
$$

The comparison between (22) and (23) is now clear: in both cases they are products of the coordinates (raised to some power) of parameterizations of the characteristic polynomial.

Choice of the parameters. Our main result is stated below; it shows that given arbitrary transition probabilities and any value of $t$ in the spectral interval, we can adjust the values of $\theta$ and $k$ so as to have the equivalence: $\widetilde{P}(z, w)=0$ if and only if $\phi(\log z, \log w)=t$.

Proposition 6. Let $p_{1}$ and $p_{2}$ be defined by (21). For any $q_{1}, q_{2}, t$ such that $q_{1}, q_{2}>0$, $q_{1}+q_{2}=\frac{1}{2}$ and $t \geqslant 1$, there exist $\theta \in(0, K)$ and $k \in[0,1)$ such that $p_{1}=q_{1}, p_{2}=q_{2}$ and $\frac{m+2\left(c_{1}+c_{2}\right)}{2\left(c_{1}+c_{2}\right)}=t$.

Proof. We first look at the equation $\frac{c_{1}}{2\left(c_{1}+c_{2}\right)}=q_{1}$. Since $c_{2}=\operatorname{sc}(K-\theta)=\frac{1}{k^{\prime} \operatorname{sc}(\theta)}$ by $[1$, 16.8.9], it is equivalent to

which results in

$$
\frac{\operatorname{sc}(\theta)}{\operatorname{sc}(\theta)+\frac{1}{k^{\prime} \operatorname{sc}(\theta)}}=2 q_{1}
$$

$$
\operatorname{sc}(\theta)=\sqrt{\frac{2 q_{1}}{1-2 q_{1}}} \frac{1}{\sqrt{k^{\prime}}},
$$

which for any $k \in[0,1)$ has a (unique) solution, see $(26)$ for an explicit expression. Now we turn to the equation involving the mass. Note first that by (12),

$$
m=2(\mathrm{~A}(\theta)+\mathrm{A}(K-\theta))-2\left(c_{1}+c_{2}\right) \text {, }
$$


and by $\left[13\right.$, Eq. (60)], $A(\theta)+A(K-\theta)=\frac{\mathrm{ns}(\theta) \mathrm{dc}(\theta)}{k^{\prime}}$, so that

$$
\frac{m+2\left(c_{1}+c_{2}\right)}{2\left(c_{1}+c_{2}\right)}=\frac{\mathrm{nc}(\theta) \mathrm{dc}(\theta)}{1+k^{\prime} \mathrm{sc}(\theta)^{2}}
$$

Let us now reproduce $[1,16.9 .3]: k^{\prime 2} \mathrm{sc}^{2}+k^{\prime 2}=k^{\prime 2} \mathrm{nc}^{2}=\mathrm{dc}^{2}-k^{2}$. These equalities allow us to derive the values of $\mathrm{nc}(\theta)$ and $\mathrm{dc}(\theta)$, starting from the value (24) of $\mathrm{sc}(\theta)$. After some computations, we conclude that

$$
\frac{m+2\left(c_{1}+c_{2}\right)}{2\left(c_{1}+c_{2}\right)}=\sqrt{1+2 q_{1}\left(1-2 q_{1}\right)\left(k^{\prime}-2+\frac{1}{k^{\prime}}\right)} .
$$

As $k$ varies in $[0,1)$, the latter function increases from 1 to $\infty$. In conclusion, $k$ is determined by solving the equation

$$
\sqrt{1+2 q_{1}\left(1-2 q_{1}\right)\left(k^{\prime}-2+\frac{1}{k^{\prime}}\right)}=t
$$

see (25), and then $\theta$ is found with (24). We can even be more explicit:

$$
\begin{aligned}
& k=\frac{t^{2}-8 q_{1}^{2}+4 q_{1}-1-\sqrt{(t-1)(t+1)\left(t+1-4 q_{1}\right)\left(t-1+4 q_{1}\right)}}{4 q_{1}\left(1-2 q_{1}\right)}, \\
& \theta=F\left(\sqrt{\frac{2 q_{1}}{k^{\prime}+2 q_{1}\left(1-k^{\prime}\right)}}, k\right)
\end{aligned}
$$

where $F$ is the incomplete elliptic integral of the first kind. The proof is completed.

The triangular lattice. The characteristic polynomial is (again by [13, Sec. 5.2])

$$
P(z, w)=m+2\left(c_{1}+c_{2}+c_{3}\right)-c_{1}\left(z+\frac{1}{z}\right)-c_{2}\left(w+\frac{1}{w}\right)-c_{3}\left(z w+\frac{1}{z w}\right),
$$

and our aim is to show an analogue of Proposition 6 . However, as the computations become much more complex in this case we will only consider here the case of uniform probabilities, corresponding to three equal conductances $c_{1}=c_{2}=c_{3}=\operatorname{sc}\left(\frac{K}{3}\right)$. By (12), the normalized characteristic polynomial takes the form

$$
\widetilde{P}(z, w)=\frac{\mathrm{A}\left(\frac{K}{3}\right)}{\mathrm{sc}\left(\frac{K}{3}\right)}-\frac{1}{6}\left(z+\frac{1}{z}\right)-\frac{1}{6}\left(w+\frac{1}{w}\right)-\frac{1}{6}\left(z w+\frac{1}{z w}\right) .
$$

Proposition 7. As $k$ varies in $[0,1), \mathrm{A}\left(\frac{K}{3}\right) / \mathrm{sc}\left(\frac{K}{3}\right)$ varies continuously in $[1, \infty)$.

Proof. Let us first prove that $t=\mathrm{A}\left(\frac{K}{3}\right) / \mathrm{sc}\left(\frac{K}{3}\right)$ is an algebraic function of $k$, solution to

$$
-27\left(1-k^{2}\right) t^{4}+18\left(1-k^{2}\right) t^{2}+2\left(2-k^{2}\right)^{2} t+1-k^{2}+k^{4}=0 .
$$

The first terms of its series expansion are

$$
t=1+\frac{3}{64} k^{4}+\frac{3}{64} k^{6}+\frac{711}{16384} k^{8}+\frac{327}{8192} k^{10}+O\left(k^{12}\right),
$$

and further computations suggest that all non-zero coefficients are positive (see Appendix $A$ for a proof). Remark that the algebraicity of $t$ is not obvious, as both functions A and sc are transcendental.

We start by showing that $s=\operatorname{sn}\left(\frac{K}{3}\right)$ is the unique power series solution to

$$
k^{2} s^{4}-2 k^{2} s^{3}+2 s-1=0 .
$$


The first terms in its expansion are

$$
s=\frac{1}{2}+\frac{3}{32} k^{2}+\frac{3}{64} k^{4}+\frac{123}{4096} k^{6}+\frac{177}{8192} k^{8}+\frac{34887}{2097152} k^{10}+O\left(k^{12}\right) .
$$

The coefficients of $s$ are again non-negative (see Appendix $A$ for a proof).

To prove (28) we start from the equality $\operatorname{sn}(K)=1$ that we rewrite as $\operatorname{sn}\left(\frac{K}{3}+\frac{2 K}{3}\right)=1$, and then we apply the classical addition formula $[1,16.17]$ together with some identities in Exercise 22 in [43, Chap. 2].

The second step is to show that both $\mathrm{A}\left(\frac{K}{3}\right)$ and $\operatorname{sc}\left(\frac{K}{3}\right)$ may be algebraically expressed in terms of $s$. By definition, $\mathrm{sc}^{2}=\left(1 / \mathrm{sn}^{2}-1\right)^{-1}$, so that $\mathrm{sc}\left(\frac{K}{3}\right)=\left(1 / s^{2}-1\right)^{-1 / 2}$. Now, using $[13,(60)$ and $(61)]$, we obtain that

$$
\mathrm{A}\left(\frac{K}{3}\right)=\frac{\sqrt{1-k^{2} s^{2}}}{3 s^{2} \sqrt{1-k^{2}}}\left(1-\frac{2\left(1-k^{2}\right) s^{4}}{1-\left(2-k^{2} s^{2}\right) s^{2}}\right) .
$$

Some computations (which we do not reproduce here) finally lead to (27).

We prove Proposition 7. Computing the discriminant of the polynomial in (27), it is easy to see that for any $k \in(0,1)$, two roots are real and the other two are non-real, complex conjugate. Moreover, the four roots are obviously non-zero. So the solution $t=t(k)$ is such that $t(0)=1$ (evaluate $(27)$ at $k=0$ ). Doing the change of variable $t \rightarrow \frac{t}{\left(1-k^{2}\right)^{1 / 3}}$ in (27), we deduce that $t(k)$ behaves as $\frac{1}{(1-k)^{1 / 3}}$ as $k \rightarrow 1$, and in particular goes to $\infty$.

\section{Minimal positive harmonic functions}

From the construction of Martin boundary, any positive harmonic function $f$ can be written as an integral of the exponential functions against some Radon measure $\mu$ supported on $2 i K^{\prime}+\mathbb{R} / 4 K \mathbb{Z}$ :

$$
\forall x \in \mathrm{G}, \quad f(x)=\int \mathrm{e}_{\left(x, x_{0}\right)}(u) \mu(\mathrm{d} u)
$$

Let us recall that positive harmonic functions are particularly important from a potential theory point of view, in relation with the concept of Doob transform. The function $f$ is then said to be minimal if we cannot find a non-trivial measure $\mu$ for this decomposition, i.e., whose support is not just a singleton; see Section 1.1.

In this section, we prove that the exponential functions $x \mapsto \mathrm{e}_{\left(x, x_{0}\right)}(u)$ with $u=2 i K^{\prime}+v$ and $v \in \mathbb{R}$ are minimal, which provides a proof of Theorem 3. This implies in particular that these exponential functions form the minimal Martin boundary of the associated killed random walk.

Recall that any isoradial graph can be viewed as a step surface in a hypercubic lattice $\mathbb{Z}^{d}$, where $d$ represents the number of possible orientations for the unit vectors representing edges of the diamond graph, and that the exponential function is naturally extended to $\mathbb{Z}^{d}$; see Sections 2.1 and 2.2.

To each point $\breve{r}=\sum_{j} n_{j} e_{j}$ in the $L^{1}$-unit ball of $\mathbb{R}^{d}$, we associate a probability measure $\nu_{\breve{r}}$ on the circle $\mathbb{R} / 4 K \mathbb{Z}$ :

$$
\nu_{\mathfrak{r}}=\sum_{j=1}^{d} n_{j}^{+} \delta_{\alpha_{j}}+n_{j}^{-} \delta_{\alpha_{j}+2 K},
$$

where $n_{j}^{ \pm}=\max \left\{ \pm n_{j}, 0\right\}$ represents the asymptotic proportion of steps $\pm e_{j}$ in a minimal path from a reference vertex $x$ to $y$, as $y$ tends to infinity in the direction $r$. Because of the monotonicity of the surface, $n_{j}^{+}$and $n_{j}^{-}$cannot be both strictly positive. Therefore, the 


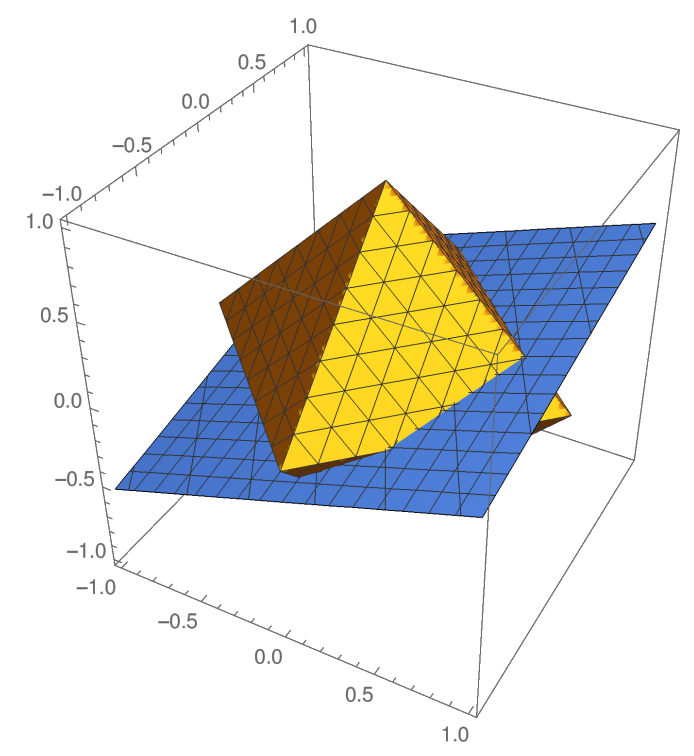

Figure 6 . The unit sphere intersected by the kernel of $\tau$ in dimension 3.

limiting measures $\nu$ have a support included in half of the circle: there is an interval of length not smaller than $2 K$ with $\nu$-measure 0 (see also Section 2.4).

Fix $u=2 i K^{\prime}+v$ with $v$ real. An important quantity of interest is the rate of growth of the massive exponential function in the asymptotic direction $r$ :

$$
\tau(\breve{r}, u)=\lim _{y \rightarrow \breve{r} \infty} \frac{1}{N} \log \left|e_{\left(x_{0}, y\right)}(u)\right|=\int_{\mathbb{R} / 4 K \mathbb{Z}} \log \left|\sqrt{k^{\prime}} \operatorname{sc}\left(\frac{u-\alpha}{2}\right)\right| \nu_{\breve{r}}(\mathrm{~d} \alpha)=\chi(v),
$$

see (15). Because $\sqrt{k^{\prime}} \operatorname{sc}(u+K)=\frac{1}{\sqrt{k^{\prime}} \operatorname{sc}(u)}$, the function $\tau$ can be rewritten as

$$
\tau(\check{\mathrm{r}}, u)=\int_{\mathbb{R} / 4 K \mathbb{Z}} \log \left|\sqrt{k^{\prime}} \mathrm{sc}\left(\frac{u-\alpha}{2}\right)\right| \tilde{\nu}_{\breve{r}}(\mathrm{~d} \alpha),
$$

where $\tilde{\nu}_{\mathfrak{r}}=\sum_{j=1}^{d} n_{j} \delta_{\alpha_{j}}$ is now a signed measure supported on the angles associated to the basis vectors $e_{1}, \ldots, e_{d}$. It is then clear that $\tau(-\breve{r}, u)=-\tau(\breve{r}, u)$. Even more, if we extend by homogeneity the definition of $\tilde{\nu}$ to all vectors of $\mathbb{R}^{d}$ by $\tilde{\nu}_{\lambda \check{r}}=\lambda \tilde{\nu}_{\breve{r}}$ for $\lambda \in \mathbb{R}$, then $\tau(\cdot, u)$ becomes a non-degenerate linear form, whose kernel is a hyperplane separating the unit sphere in two parts; see Figure 6 for an example.

Lemma 8. The $L^{1}$-unit sphere is separated in two (connected) hemispheres, corresponding to positive and negative values of $\tau(\cdot, u)$.

Informally, this lemma says that for any $u$ in the torus, $\mathrm{e}_{\left(x_{0}, y\right)}$ tends exponentially fast to 0 for half of the directions to infinity. This is in particular true for the positive exponential functions for which $u=2 i K^{\prime}+v$, with $v \in \mathbb{R}$. We claim that this property is characterizing the massive exponential functions for $u=2 i K^{\prime}+v$ with $v$ real, among all positive massive harmonic functions:

Proposition 9. If $h$ is a positive harmonic function which tends to 0 in at least half of the directions, then it is proportional to an exponential function. 


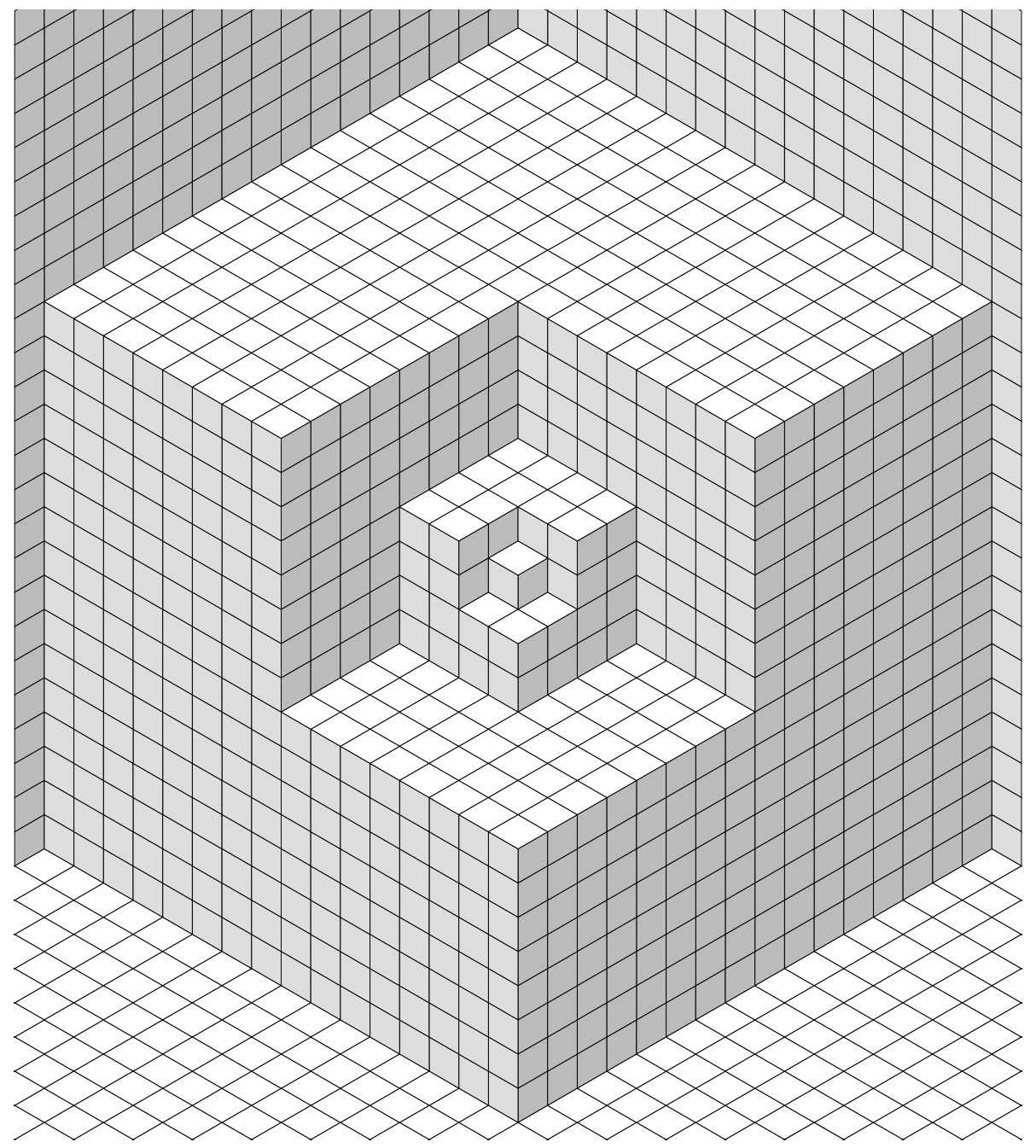

Figure 7. A piece of the diamond graph of an isoradial graph which is not asymptotically flat. From the center, there are "waves" of size growing exponentially. When going to infinity in a direction in the plane, the proportions of the different types of steps do not converge, but instead oscillate.

Proof. Write $h$ as an integral over the positive exponential function for some positive measure $\mu$ as in (29). Therefore, the set of directions along which $h$ goes to infinity is the union of the half-spaces for which $\mathrm{e}_{\left(x_{0}, y\right)}(u)$ goes to infinity, for $u$ in the support of $\mu$. If $\mu$ is non-trivial then this is strictly more than a half-space, which is impossible by hypothesis. Therefore $\mu$ should have a support reduced to a singleton, and $h$ is proportional to an exponential function.

Proof of Theorem 3. If $h$ is less or equal to $h_{v}$, then it goes to 0 at least in half of the directions (the ones where $h_{v}$ itself goes to 0 ). We can therefore apply Proposition 9 to conclude. 
Appendix A. Some thoughts on a positivity question (by Alin Bostan)

We consider the following question: let $s(k)=\sum_{n \geqslant 0} s_{n} k^{n}$, starting

$$
s(k)=\frac{1}{2}+\frac{3}{32} k^{2}+\frac{3}{64} k^{4}+\frac{123}{4096} k^{6}+\frac{177}{8192} k^{8}+\frac{34887}{2097152} k^{10}+\cdots,
$$

be the unique power series solution of the algebraic equation

$$
k^{2} s^{4}-2 k^{2} s^{3}+2 s-1=0 .
$$

We want to prove that all the coefficients of $s(k)$ are non-negative, and more precisely that all the coefficients $s_{2 n}$ are positive.

Note that the sequence $\left(s_{n}\right)_{n \geqslant 0}$ satisfies various types of recurrences, either non-linear, obtained by extracting the coefficient of $k^{n}$ in both sides of (30), or even linear, obtained by using a linear differential equation (with polynomial coefficients in $k$ ) satisfied by $s(k)$. However, these recurrences cannot be used directly to solve our positivity question. For instance, from the algebraic equation (30), Cockle's algorithm (see [8] and the references therein) proves that $s(k)$ satisfies the $3 r d$ order linear inhomogeneous differential equation

$$
\begin{aligned}
9 k^{3}(k-1)^{2}(k+1)^{2} & s^{\prime \prime \prime}(k)+9 k^{2}(k-1)(k+1)\left(5 k^{2}-1\right) s^{\prime \prime}(k) \\
& +k\left(35 k^{4}-14 k^{2}-13\right) s^{\prime}(k)-4\left(k^{2}-2\right) s(k)=4-2 k^{2},
\end{aligned}
$$

from which a coefficient extraction proves that $\left(s_{n}\right)_{n \geqslant 0}$ satisfies the linear recurrence

$$
\begin{aligned}
(3 n+10)(3 n+14)(n+2) s_{n+4}+n(3 n+2) & (3 n+4) s_{n} \\
= & 2\left(9 n^{3}+54 n^{2}+106 n+70\right) s_{n+2} .
\end{aligned}
$$

It is not obvious from this recurrence how to derive a non-negativity proof because of the plus sign in front of the coefficient of $s_{n}$. In principle, two quite general methods might be applied to this kind of question (alone, or combined): one relying on (effective) asymptotics for the coefficients of algebraic functions [24, p. 504-505], the other relying on the approach of $[38, \S 4.2]$. Instead, we present two different proofs.

The first one is based on the following hypergeometric expression for $s(k)$ :

$$
s(k)=\frac{1}{2}+\frac{3}{2} \cdot\left(\frac{k}{4} \cdot{ }_{2} F_{1}\left[\begin{array}{cc}
\frac{1}{2} & \frac{5}{6} \\
\frac{5}{3} & ; k^{2}
\end{array}\right]\right)^{2}
$$

where the Gauss' hypergeometric series with parameters $\frac{1}{2}, \frac{5}{6}$ and $\frac{5}{3}$ is defined by

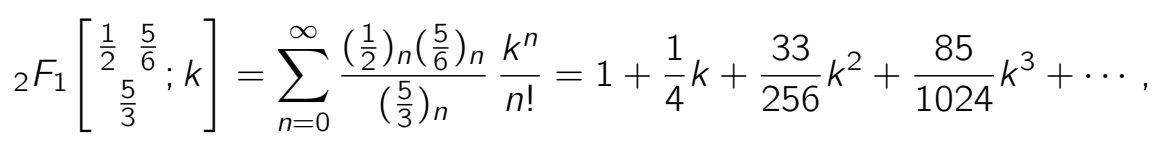

and $(x)_{n}$ denotes the Pochhammer symbol $(x)_{n}=x(x+1) \cdots(x+n-1)$ for $n \in \mathbb{N}$.

Once found (e.g., using algorithmic tools for automated guessing [37], or for differential equation solving [29]), equality (33) can easily be proved using closure properties of D-finite functions [53]: from the 2 nd order linear differential equation satisfied by ${ }_{2} F_{1}\left[\frac{1}{2} \frac{\frac{5}{6}}{3} ; k\right]$, one computes a 3rd order differential equation satisfied by the right-hand side of (33), which appears to coincide with the differential equation (31) satisfied by the left-hand side of (33). Therefore, the coefficients sequences of both right-hand side and left-hand side of (33) satisfy the recurrence relation (32), hence they are equal since their initial terms coincide. 
A shorter proof of equality (33), with a different (more geometric) flavor, goes as follows: on the one hand, the hypergeometric function in (34) satisfies the algebraic transformation ${ }^{4}$

$$
{ }_{2} F_{1}\left[\begin{array}{c}
\frac{1}{2} \frac{5}{6} \\
\frac{5}{3}
\end{array} ; \frac{x(x+2)^{3}}{(2 x+1)^{3}}\right]=4 \frac{(2 x+1)^{\frac{3}{2}}}{(x+2)^{2}} .
$$

On the other hand, it is easy to prove, starting from the polynomial (30), that the algebraic function $s(k)$ satisfies

$$
s\left(\frac{x^{\frac{1}{2}}(x+2)^{\frac{3}{2}}}{(2 x+1)^{\frac{3}{2}}}\right)=\frac{2 x+1}{x+2} .
$$

Denoting $\varphi(x)=x(x+2)^{3} /(2 x+1)^{3}$, identity (33) becomes trivial in the new variable $x$ :

$$
\frac{2 x+1}{x+2}=\frac{1}{2}+\frac{3}{2} \cdot \varphi(x) \cdot\left(\frac{(2 x+1)^{3 / 2}}{(x+2)^{2}}\right)^{2} \text {. }
$$

Now that (33) is proved, the positivity of the coefficients $s_{2 n}$ of $s(k)$ is a direct consequence of the obvious fact that the hypergeometric power series in (34) has positive coefficients.

The second proof is based on the so-called Stieltjes inversion formula [48, Lecture 2], that allows to express in some cases the $n$-th term of a sequence as the $n$-th moment of a positive density. Using this formula, one can deduce the following expression:

$$
s_{2 n}=\frac{\sqrt{3}}{\pi} \cdot \int_{0}^{1} k^{n} \cdot H_{1}(k) \cdot\left(\frac{1}{2^{4 / 3}} \cdot \frac{H_{2}(k)}{k^{2 / 3}}-\frac{1}{2^{8 / 3}} \cdot \frac{H_{1}(k)}{k^{1 / 3}}\right) d k
$$

where $H_{1}(k)$ and $H_{2}(k)$ are the hypergeometric functions

$$
H_{1}={ }_{2} F_{1}\left[\begin{array}{c}
\frac{1}{6} \frac{5}{6} \\
\frac{4}{3}
\end{array} ; k\right]=1+\frac{5}{48} k+\cdots, \quad H_{2}={ }_{2} F_{1}\left[\begin{array}{c}
-\frac{1}{6} \\
\frac{2}{2} \\
\frac{2}{3}
\end{array} ; k\right]=1-\frac{1}{8} k-\frac{3}{64} k^{2}-\cdots .
$$

At this point, positivity is not yet apparent. However, using the change of variables $k=\varphi(x)$, and using the fact that $H_{1}(\varphi(x))$ and $H_{2}(\varphi(x))$ become the simple algebraic functions $2(x+1)^{1 / 3}(2 x+1)^{1 / 2} /(x+2)$ and $(2 x+1)^{-1 / 2}$, the previous expression simplifies to:

$$
s_{2 n}=\frac{\sqrt{3} \cdot \sqrt[3]{2}}{\pi} \cdot \int_{0}^{1} \frac{(x-1)^{2}\left(\sqrt[3]{2}-\sqrt[3]{x^{2}+x}\right) \sqrt[3]{x+1}}{(x+2)(2 x+1)^{2} x^{2 / 3}} \varphi(x)^{n} \mathrm{~d} x
$$

Once found, equality (35) can again be proved automatically using algorithmic tools, notably the method of "creative telescoping" [2, 41, 10].

Note that Lagrange inversion is a tempting alternative to the previous moment approach; however, in our case, it gives that for all $n>0$,

$$
s_{2 n}=\frac{1}{2 n} \cdot\left[z^{-1}\right]\left(\frac{(z+1)^{3}(3-z)}{16 z}\right)^{n}=\frac{(-1)^{n}}{n \cdot 2^{4 n+1}} \cdot \sum_{i=0}^{n}\left(\begin{array}{c}
3 n \\
i
\end{array}\right)\left(\begin{array}{c}
n \\
i+1
\end{array}\right)(-3)^{i+1},
$$

and positivity is not clear on any of these expressions.

In contrast, identity (35) obviously proves that the sequence $\left(s_{2 n}\right)_{n \geqslant 0}$ is positive. And it actually proves much more, namely that $\left(s_{2 n}\right)_{n \geqslant 0}$ is a Stieltjes-Hausdorff moment sequence, and in particular that it is log-convex, i.e., $s_{2 n+2} s_{2 n-2} \geqslant s_{2 n}^{2}$ for all $n \geqslant 1$ [51, §2] and $\Delta^{k} s_{2 n} \geqslant 0$ for all $k \geqslant 0\left[52\right.$, p. 338], where $\Delta$ is the difference operator $\Delta\left(c_{n}\right)=\left(c_{n}-c_{n+1}\right)$.

\footnotetext{
${ }^{4}$ This is inspired by the Darboux covering for tetrahedral hypergeometric equations of the Schwarz type $(1 / 3,1 / 3,2 / 3)[54, \S 6.1]$, see also [55]. Note that the same change of variables has been used in $[9, \S 4]$.
} 
We also consider the following related question: let $t(k)=\sum_{n \geqslant 0} t_{n} k^{n}$, starting

$$
t(k)=1+\frac{3}{64} k^{4}+\frac{3}{64} k^{6}+\frac{711}{16384} k^{8}+\frac{327}{8192} k^{10}+\cdots,
$$

be the unique power series solution of the algebraic equation

$$
-27\left(1-k^{2}\right) t^{4}+18\left(1-k^{2}\right) t^{2}+2\left(2-k^{2}\right)^{2} t+1-k^{2}+k^{4}=0 .
$$

We want to prove that all the coefficients $t_{n}$ are non-negative.

First, we remark that $t\left(\varphi(x)^{\frac{1}{2}}\right)=\left(x^{2}+x+1\right) /\left(1+x-2 x^{2}\right)$. Since $s\left(\varphi(x)^{\frac{1}{2}}\right)=$ $(2 x+1) /(x+2)$, this implies that $t(k)=R(s(k))$, where $R$ is the rational function

$$
R(z)=\frac{z^{2}-z+1}{3 z(1-z)}
$$

As a consequence of this and of identity (33), we get that

$$
t(k)=\left(1+3 H^{4}\right) /\left(1-9 H^{4}\right)=\left(1+3 H^{4}\right) \cdot \sum_{n \geqslant 0}\left(9 H^{4}\right)^{n},
$$

where $H$ is the hypergeometric function with non-negative coefficients

$$
H=\frac{k}{4} \cdot{ }_{2} F_{1}\left[\begin{array}{c}
\frac{1}{2} \frac{5}{6} \\
\frac{5}{3}
\end{array} ; k^{2}\right]=\frac{1}{4} k+\frac{1}{16} k^{3}+\frac{33}{1024} k^{5}+\cdots,
$$

therefore all the coefficients of $t(k)$ are non-negative.

\section{References}

[1] Abramowitz, M. and Stegun, I. A. Handbook of mathematical functions with formulas, graphs, and mathematical tables. National Bureau of Standards Applied Mathematics Series, 55 (1964)

[2] Almkvist, G. and Zeilberger, D. The method of differentiating under the integral sign. J. Symbolic Comput. 10 (1990) 571-591

[3] Babillot, M. Théorie du renouvellement pour des chaînes semi-markoviennes transientes. Ann. Inst. H. Poincaré Probab. Statist. 24 (1988) 507-569

[4] Beffara, V., Duminil-Copin, H. and Smirnov, S. On the critical parameters of the $q \leqslant 4$ random-cluster model on isoradial graphs. J. Phys. A 48 (2015) 484003, 25 pp.

[5] Biane, P. Théorème de Ney-Spitzer sur le dual de SU(2). Trans. Amer. Math. Soc. 345 (1994) 179-194

[6] Bobenko, A. I. and Suris, Y. B. Discrete differential geometry. Integrable structure. Graduate Studies in Mathematics, 98. American Mathematical Society, Providence, RI (2008)

[7] Bodini, O., Fernique, T. and Rémila, É. A characterization of flip-accessibility for rhombus tilings of the whole plane. Inform. and Comput. 206 (2008) 1065-1073

[8] Bostan, A., Chyzak, F., Salvy, B., Lecerf, G. and Schost, É. Differential equations for algebraic functions. Proceedings of the 2007 International Symposium on Symbolic and Algebraic Computation (2007) 25-32

[9] Bostan, A., Kurkova, I. and Raschel, K. A human proof of Gessel's lattice path conjecture. Trans. Amer. Math. Soc. 369 (2017) 1365-1393

[10] Bostan, A., Dumont, L. and Salvy, B. Efficient algorithms for mixed creative telescoping. Proceedings of the 2016 International Symposium on Symbolic and Algebraic Computation (2016) 127-134

[11] Boutillier, C. and de Tilière, B. The critical Z-invariant Ising model via dimers: locality property. Comm. Math. Phys. 301 (2011) 473-516

[12] Boutillier, C. and de Tilière, B. Statistical mechanics on isoradial graphs. Deuschel, J.-D. (ed.) et al., Probability in complex physical systems. Springer Proceedings in Mathematics 11 (2012) 491-512

[13] Boutillier, C., de Tilière, B. and Raschel, K. The Z-invariant massive Laplacian on isoradial graphs. Invent. Math. 208 (2017) 109-189

[14] Boutillier, C., de Tilière, B. and Raschel, K. The Z-invariant Ising model via dimers. Probab. Theory Related Fields, 174 (2019) 235-305

[15] de Bruijn, N. G. Algebraic theory of Penrose's nonperiodic tilings of the plane. I. Nederl. Akad. Wetensch. Indag. Math. 43 (1981) 39-52 
[16] Chelkak, D. and Smirnov, S. Discrete complex analysis on isoradial graphs. Adv. Math. 228 (2011) 1590-1630

[17] Choquet, G. and Deny, J. Sur l'équation de convolution $\mu=\mu * \sigma$. C. R. Acad. Sci. Paris 250 (1960) 799-801

[18] de Tilière, B. Scaling limit of isoradial dimer models and the case of triangular quadri-tilings. Ann. Inst. H. Poincaré Sect. B. 43 (2007) 729-750

[19] Doob, J. L. Discrete potential theory and boundaries. J. Math. Mech. 8 (1959) 433-458

[20] Doob, J. L., Snell, J. L. and Williamson, R. E. Application of boundary theory to sums of independent random variables. Contributions to probability and statistics (1960) 182-197 Stanford Univ. Press, Stanford

[21] Duffin, R. J. Potential theory on a rhombic lattice. J. Combinatorial Theory 5 (1968), 258-272

[22] Duminil-Copin, H., Li, J.-H. and Manolescu, I. Universality for the random-cluster model on isoradial graphs. Electron. J. Probab. 23 (2018) Paper No. 96, 70pp.

[23] Dussaule, M. The Martin boundary of a free product of abelian groups. Ann. Inst. Fourier (Grenoble) 70 (2020), 313-373

[24] Flajolet, P. and Sedgewick, R. Analytic combinatorics. Cambridge University Press (2009)

[25] Gelfand, I. M., Kapranov, M. M. and Zelevinsky, A. V. Discriminants, Resultants, and Multidimensional Determinants. Birkhäuser (1994)

[26] George, T. Spectra of biperiodic planar networks. Preprint arXiv:1901.06353 (2019)

[27] Godefroy, L. Martin boundary of polynomial random walks on the $d$-dimensional lattice of nonnegative integers. J. Theoret. Probab. 17 (2004) 111-129

[28] Grimmett, G. R. and Manolescu, I. Bond percolation on isoradial graphs: criticality and universality. Probab. Theory Related Fields, 159 (2014) 273-327

[29] Imamoglu, E. and van Hoeij, M. Computing hypergeometric solutions of second order linear differential equations using quotients of formal solutions and integral bases. J. Symbolic Comput. 83 (2017) 254-271

[30] Hennequin, P.-L. Processus de Markoff en cascade. Ann. Inst. H. Poincaré 18 (1963) 109-195

[31] Hunt, G. A. Markoff chains and Martin boundaries. Illinois J. Math. 4 (1960) 313-340

[32] Ignatiouk-Robert, I. and Loree, C. Martin boundary of a killed random walk on a quadrant. Ann. Probab. 38 (2010) 1106-1142

[33] Ignatiouk-Robert, I. Martin boundary of a killed random walk on a half-space. J. Theoret. Probab. 21 (2008) 35-68

[34] Ignatiouk-Robert, I. Martin boundary of a reflected random walk on a half-space. Probab. Theory Related Fields 148 (2010) 197-245

[35] Ignatiouk-Robert, I. t-Martin boundary of reflected random walks on a half-space. Electron. Commun. Probab. 15 (2010) 149-161

[36] Ignatiouk-Robert, I. Harmonic functions of random walks in a semigroup via ladder heights. J. Theoret. Probab. (to appear)

[37] Kauers, M. The Guess-and-Prove Paradigm in Action. Internationale Mathematische Nachrichten 237 (2018) 1-15

[38] Kauers, M. and Pillwein, V. When can we detect that a P-finite sequence is positive? Proceedings of the 2010 International Symposium on Symbolic and Algebraic Computation (2010) 195-201

[39] Kenyon, R. The Laplacian and Dirac operators on critical planar graphs. Invent. Math. 150 (2002) 409-439

[40] Kenyon, R. Determinantal spanning forests on planar graphs Ann. Probab. 47 (2019) 952-988

[41] Koutschan, C. Creative telescoping for holonomic functions. Computer algebra in quantum field theory. Texts Monogr. Symbol. Comput. (2013) 171-194

[42] Kurkova, I. A. and Malyshev, V. A. Martin boundary and elliptic curves. Markov Process. Related Fields 4 (1998) 203-272

[43] Lawden, D. Elliptic functions and applications. Applied Mathematical Sciences, 80. Springer-Verlag, New York (1989)

[44] Mercat, C. Discrete Riemann surfaces and the Ising model. Comm. Math. Phys. 218 (2001) 177-216

[45] Martin, R. S. Minimal positive harmonic functions. Trans. Amer. Math. Soc. 49 (1941) 137-172

[46] Mustapha, S. Gaussian estimates for spatially inhomogeneous random walks on $\mathbb{Z}^{d}$. Ann. Probab. 34 (2006) 264-283

[47] Ney, P. and Spitzer, F. The Martin boundary for random walk. Trans. Amer. Math. Soc. 121 (1966) 116-132 
[48] Nica, A. and Speicher, R. Lectures on the combinatorics of free probability. London Mathematical Society Lecture Note Series, 335. Cambridge University Press (2006)

[49] Pemantle, R. and Wilson, M. Analytic Combinatorics in Several Variables. Cambridge Studies in Advanced Mathematics (2013)

[50] Sawyer, S. A. Martin boundaries and random walks. Harmonic functions on trees and buildings (New York, 1995), 17-44, Contemp. Math., 206, Amer. Math. Soc., Providence, RI (1997)

[51] Sokal, A. D. The Euler and Springer numbers as moment sequences. Expo. Math. 38 (2020), 1-26

[52] Spitzer, F. Principles of random walk. The University Series in Higher Mathematics D. Van Nostrand Co., Inc., Princeton, N.J.-Toronto-London (1964)

[53] Stanley, R. P. Differentiably finite power series. European J. Combin. 1 (1980) 175-188

[54] Vidūnas, R. Transformations of algebraic Gauss hypergeometric functions. Preprint arXiv:0807.4808 (2008)

[55] Vidūnas, R. Algebraic transformations of Gauss hypergeometric functions. Funkcial. Ekvac. 52 139-180 (2009)

[56] Woess, W. Random walks on infinite graphs and groups. Cambridge Tracts in Mathematics, 138. Cambridge University Press, Cambridge (2000)

Email address: alin.bostan@inria.fr

Email address: cedric.boutillier@sorbonne-universite.fr

Email address: raschel@math.cnrs.fr 